\title{
On the First Initial-Boundary Value Problem of the Generalized Burgers' Equation
}

\author{
By \\ Atusi TANI*
}

\section{$\S 1$. Introduction and Notations}

E. Hopf discussed in details on the Cauchy problem of Burgers' equation in his famous paper [5]. Since then, many papers on the equation and its related topics have been published. However, they have not treated the initial-boundary value problem of it. The author previously discussed on the first initial-boundary value problem of this equation in [17]. Recently, N. Itaya has shown the existence and the uniqueness, in a certain sense, of the temporally global solution of the Cauchy problem of the following generalized Burgers' equation:

$$
\begin{aligned}
& (1.1)^{1} \\
& (1.1)^{2}
\end{aligned}\left\{\begin{array}{l}
\frac{\partial v}{\partial t}(x, t)=\frac{\mu}{\rho(x, t)} \frac{\partial^{2}}{\partial x^{2}} v(x, t)-v(x, t) \frac{\partial}{\partial x} v(x, t), \\
\frac{\partial \rho}{\partial t}+\frac{\partial}{\partial x}(\rho v)=0, \quad(\mu \text { is a positive constant })
\end{array}\right.
$$

in [11] (cf. [9], [10]). Stimulated by his work, the author attempts to discuss on the first initial-boundary value problem of (1.1) in $[0, X] \subset$ $R^{1}$, especially from the view-point of the temporally global behavior of the solution of (1.1).

The author expresses his hearty thanks to Professor N. Itaya, of Kôbe College of Commerce, whose stimulating guidance and encouragement with kind discussions have meant so much to him throughout his work in the present paper, and to Professors T. Ugaheri and M. Nagasawa who have given many valuable suggestions and constantly encouraged him to write up this paper.

Communicated by S. Matsuura, November 21, 1973.

* Department of Applied Physics, Tokyo Institute of Technology, Tokyo. 
Notations. The functions considered in this paper should be understood to be defined in $[0, X]$ or $[0, X] \times[0, T](0<X<+\infty, 0<T<+\infty)$ and continuously differentiable as many times as necessary.

$$
\begin{aligned}
& \left(\Omega=(0, X), \bar{\Omega}=[0, X], S_{T}^{0}=\{0\} \times[0, T],\right. \\
& S_{T}^{X}=\{X\} \times[0, \mathrm{~T}], S_{T}=S_{T}^{0} \cup S_{T}^{X}, \Gamma_{T}=S_{T} \cup \Omega \times\{0\}, \\
& Q_{T}=\Omega \times(0, T), \bar{Q}_{T}=\bar{\Omega} \times[0, T] \\
& \left\{\begin{array}{l}
|u(x)|^{(0)} \equiv \sup _{\bar{\Omega}}|u(x)|, \\
|u(x)|^{(\alpha)} \equiv \sup _{\bar{\Omega}, x \neq x^{\prime}} \frac{\left|u(x)-u\left(x^{\prime}\right)\right|}{\left|x-x^{\prime}\right|^{\alpha}} .
\end{array}\right. \\
& \left\{\begin{array}{l}
\|u(x)\|^{(n)} \equiv \sum_{i=0}^{n}\left|D_{x}^{i} u(x)\right|^{(0)}, \\
\|u(x)\|^{(n+\alpha)} \equiv\|u(x)\|^{(n)}+\left|D_{x}^{n} u(x)\right|^{(\alpha)} \quad(n=0,1, \ldots) .
\end{array}\right. \\
& \left(|v(x, t)|_{T}^{(0)} \equiv \sup _{\bar{Q}_{T}}|v(x, t)|\right. \\
& \begin{array}{l}
|v(x, t)|_{x, T}^{(\alpha)} \equiv \sup _{\bar{Q}_{T}, x \neq x^{\prime}} \frac{\left|v(x, t)-v\left(x^{\prime}, t\right)\right|}{\left|x-x^{\prime}\right|^{\alpha}} \\
|v(x, t)|_{t, T}^{(\alpha / 2)} \equiv \sup _{\bar{Q}_{T}, t \neq t^{\prime}} \frac{\left|v(x, t)-v\left(x, t^{\prime}\right)\right|}{\left|t-t^{\prime}\right|^{\alpha / 2}}
\end{array} \\
& \left.|| v(x, t)\right|_{T} ^{(\alpha)} \equiv|v(x, t)|_{x, T}^{(\alpha)}+|v(x, t)|_{t, T}^{(\alpha / 2)} . \\
& \int\|v\|_{T}^{(n)} \equiv \sum_{2 r+s=0}^{n}\left|D_{t}^{r} D_{x}^{s} v\right|_{T}^{(0)} \\
& \|v\|_{T}^{(n+\alpha)} \equiv\|v\|_{T}^{(n)}+\sum_{2 r+s=n}\left|D_{t}^{r} D_{x}^{s} v\right|_{x, T}^{(\alpha)}+ \\
& +\sum_{2 r+s=(n-1) \vee 0}^{n}\left|D_{t}^{r} D_{x}^{s} v\right|_{t, T}^{(\alpha / 2)},(a \vee b \equiv \max (a, b)), \\
& <v>_{T}^{(n, \alpha)} \equiv \sum_{s=0}^{n}\left|D_{x}^{s} v\right|_{T}^{(0)}+\sum_{s=0}^{n-1}\left|D_{x}^{s} v\right|_{t, T}^{(\alpha / 2)} \\
& { }^{\prime}<v>{ }_{T}^{(n, \alpha)} \equiv\left|D_{x}^{n} v\right|_{T}^{(\alpha)}, \\
& \ll v \gg{ }_{T}^{(n, \alpha)} \equiv\langle v\rangle_{T}^{(n, \alpha)}+{ }^{\prime}\langle v\rangle_{T}^{(n, \alpha)},
\end{aligned}
$$


where $r$ and $s$ are non-negative integers.

$$
\left\{\begin{array}{l}
H^{n} \equiv\left\{u(x) \mid\|u\|^{(n)}<+\infty\right\}, \\
H^{n+\alpha} \equiv\left\{u(x) \mid\|u\|^{(n+\alpha)}<+\infty\right\}, \\
H_{T}^{n+\alpha} \equiv\left\{v(x, t) \mid\|v\|_{T}^{(n+\alpha)}<+\infty\right\}, \\
\hat{H}_{T}^{n+\alpha} \equiv\left\{v(x, t) \mid \ll v \gg_{T}^{(n+\alpha)}<+\infty\right\}, \\
B_{T}^{n} \equiv\left\{v(x, t) \sum_{r+s=0}^{n}\left|D_{t}^{r} D_{x}^{s} v\right|_{T}^{(0)}<+\infty\right\}, \\
B_{T}^{n+\alpha} \equiv\left\{\left.v(x, t)\left|\sum_{r+s=0}^{n}\right| D_{t}^{r} D_{x}^{s} v\right|_{T} ^{(0)}+\sum_{r+s=n}\left|D_{t}^{r} D_{x}^{s} v\right|_{T}^{(\alpha)}<+\infty\right\} .
\end{array}\right.
$$

Other notations, not described above, will be explained where they appear.

\section{§2. Preliminaries}

We assume for (1.1) the following initial-boundary conditions:

$$
\begin{gathered}
v(x, 0)=v_{0}(x) \in H^{2+\alpha}, \quad \rho(x, 0)=\rho_{0}(x) \in H^{1}, \\
\left(0<\bar{\rho}_{0} \equiv \inf _{\bar{\Omega}} \rho_{0}(x) \leq \rho_{0}(x) \leq \bar{\rho}_{0} \equiv\left|\rho_{0}(x)\right|^{(0)}\right) \\
v(0, t)=v(X, t)=0,
\end{gathered}
$$

and for $v(x, t)$ the following compatible condition:

$$
v_{x x}(x, t)_{\mid S_{T}^{0}}=v_{x x}(x, t)_{\mid S_{T}^{x}}=0
$$

Let $(v, \rho)$ be a solution in $H_{T}^{2+\alpha} \times B_{T}^{1}$ of (1.1) satisfying the initialboundary conditions (2.1) and (2.2), and $\bar{x}(\tau ; x, t)$ be the solution curve of the characteristic equation for $(1.1)^{2}$ as a linear equation in $\rho$ :

$$
\left\{\begin{array}{l}
\frac{d}{d \tau} \bar{x}(\tau ; x, t)=v(\bar{x}(\tau ; x, t), \tau) \quad(0 \leqq \tau \leqq t \leqq T) \\
\bar{x}(t ; x, t)=x
\end{array}\right.
$$

Since $v \in H_{T}^{2+\alpha}$, the solution curve for (2.4) starting at an arbitrary point 
$(x, t) \in \bar{Q}_{T}$ is unique. By (2.4) we have

$$
\bar{x}_{x}(\tau ; x, t)=\frac{\partial \bar{x}}{\partial x}(\tau ; x, t)=\exp \left\{-\int_{\tau}^{t} v_{x}\left(\bar{x}\left(\tau^{\prime} ; x, t\right), \tau^{\prime}\right) d \tau^{\prime}\right\}
$$

If $v(x, t) \in H_{T}^{2+\alpha}$ is given in $(1.1)^{2}$, then $\rho(x, t)$ or $\rho_{v}(x, t)$ is uniquely determined by the formula:

$$
\begin{aligned}
\rho(x, t) & \equiv \rho_{v}(x, t)=\rho_{0}(\bar{x}(0 ; x, t)) \bar{x}_{x}(0 ; x, t) \\
& =\rho_{0}(\bar{x}(0 ; x, t)) \exp \left\{-\int_{0}^{t} v_{x}\left(\bar{x}\left(\tau^{\prime} ; x, t\right), \tau^{\prime}\right) d \tau^{\prime}\right\}
\end{aligned}
$$

For simplicity, we put

$$
\left\{\begin{array}{l}
\bar{\rho}(\tau ; x, t)=\rho(\bar{x}(\tau ; x, t), \tau), \\
\bar{v}(\tau ; x, t)=v(\bar{x}(\tau ; x, t), \tau), \text { etc. }
\end{array}\right.
$$

By $(1.1)^{2}$, (2.4) and (2.6), the following fundamental lemma holds (cf. [11]).

Lemma 2.1. If $(v, \rho)$ is a solution of (1.1) in $H_{T}^{2+\alpha} \times B_{T}^{1}$ with (2.1) and (2.2), then the following equation holds:

$$
\mu \int_{0}^{t} \bar{v}_{x x}(\tau ; x, t) \bar{x}_{x}(\tau ; x, t) d \tau=\rho(x, t)\left\{v(x, t)-v_{0}\left(x_{0}(x, t)\right)\right\}
$$

where

$$
x_{0}(x, t)=\bar{x}(0 ; x, t)
$$

Concerning $\rho(x, t)$, we have, by (2.6) and simple calculations,

Lemma 2.2. If $\left(v, \rho_{v}\right)$ and $\left(w, \rho_{w}\right)$ are solutions of (1.1) with (2.1) and (2.2) in $H_{T}^{2+\alpha} \times B_{T}^{1}$ (cf. (2.6)). then

$$
\left|\frac{1}{\rho_{v}}-\frac{1}{\rho_{w}}\right|_{T_{0}}^{(0)} \leq C_{1}\left(T_{0} ; v, w\right)\|v-w\|_{T_{0}}^{(1)}\left(0 \leqq T_{0} \leqq T\right),
$$

where $C_{1}\left(T_{0} ; v, w\right) \downarrow 0$ as $T_{0} \downarrow 0$.

From (2.6), $(v, \rho) \in H_{T}^{2+\alpha} \times B_{T}^{1}$ implies $\mu / \rho \in H_{T}^{\alpha}$.

Let 
(2.11)

$$
\begin{aligned}
& Z^{0}(x-\xi, t ; \xi, \tau ; \mu / \rho)=\frac{1}{2 \sqrt{\pi}}(\mu / \rho(\xi, \tau))^{-1 / 2}(t-\tau)^{-1 / 2} \times \\
& \times \exp \left\{-(x-\xi)^{2} / 4 \frac{\mu}{\rho}(\xi, \tau)(t-\tau)\right\} \quad(0 \leqq \tau \leqq t \leqq T)
\end{aligned}
$$

be the parametrix of the linear parabolic equiaton:

$$
\frac{\partial w}{\partial t}(x, t)=\frac{\mu}{\rho}(\xi, \tau) \frac{\partial^{2} w}{\partial x^{2}}(x, t)
$$

Then the parametrix of (2.12) with (2.2) is given by

$$
\begin{aligned}
Z(x-\xi, t ; \xi, \tau ; \mu / \rho)= & \sum_{n=-\infty}^{\infty}\left\{Z^{0}(x-\xi+2 n X, t ; \xi, \tau ; \mu / \rho)-\right. \\
& \left.-Z^{0}(x+\xi+2 n X, t ; \xi, \tau ; \mu / \rho)\right\}
\end{aligned}
$$

As is well known, $Z^{0}$ has the following properties:

\section{Lemma 2.3.}

(i)

$$
\begin{aligned}
&\left|D_{x}^{m} Z^{0}(x-\xi, t ; \xi, \tau ; \mu / \rho)\right| \leqq C_{2}^{(m)}(t-\tau)^{-\frac{1+m}{2}} \times \\
& \times \exp \left\{-(x-\xi)^{2} / 8\left|\frac{\rho}{\mu}\right|_{T}^{(0)}(t-\tau)\right\},
\end{aligned}
$$

(ii) for $t>t^{\prime}>\tau$,

$$
\begin{aligned}
&\left|D_{x}^{m} Z^{0}(x-\xi, t ; \xi, \tau ; \mu / \rho)-D_{x}^{m} Z^{0}\left(x-\xi, t^{\prime} ; \xi, \tau ; \mu / \rho\right)\right| \leqq C_{3}^{(m)}\left(t-t^{\prime}\right) \times \\
& \times\left(t^{\prime}-\tau\right)^{-\frac{3+m}{2}} \exp \left\{-(x-\xi)^{2} / 8\left|\frac{\mu}{\rho}\right|_{T}^{(0)}(t-\tau)\right\},
\end{aligned}
$$

(iii) $\quad\left|D_{x}^{m} Z^{0}(x-\xi, t ; \xi, \tau ; \mu / \rho)-D_{x^{\prime}}^{m} Z^{0}\left(x^{\prime}-\xi, t ; \xi, \tau ; \mu / \rho\right)\right| \leqq C_{4}^{(m)}\left|x-x^{\prime}\right| \times$

$$
\times(t-\tau)^{-\frac{2+m}{2}} \exp \left\{-\left(x^{\prime \prime}-\xi\right)^{2} / 8\left|\frac{\mu}{\rho}\right|_{T}^{(0)}(t-\tau)\right\},
$$

where

$$
x^{\prime \prime}= \begin{cases}x & \left(\text { if }|x-\xi|<\left|x^{\prime}-\xi\right|\right) \\ x^{\prime} & \text { (otherwise) }\end{cases}
$$

(iv) $\quad\left|D_{t}^{k} D_{z}^{m} Z^{0}(z, t ; \xi, \tau ; \mu / \rho)-D_{t}^{k} D_{z}^{m} Z^{0}\left(z, t ; \xi^{\prime}, \tau ; \mu / \rho\right)\right| \leqq C_{5}^{(k, m)}\left|\xi-\xi^{\prime}\right|^{\alpha} \times$

$$
\times(t-\tau)^{-\frac{1+m+2 k}{2}} \exp \left\{-z^{2} / 8\left|\frac{\mu}{\rho}\right|_{T}^{(0)}(t-\tau)\right\} .
$$


Using the relation (2.13) and Lemma 2.3, $Z$ is estimated as follows:

Lemma 2.4.

$$
\begin{aligned}
\left|D_{x}^{m} Z(x-\xi, t ; \xi, \tau ; \mu / \rho)\right| \leqq C_{6}^{(m)}(t-\tau)^{-\frac{1+m}{2}} \times \\
\times \exp \left\{-(x-\xi)^{2} / 16\left|\frac{\mu}{\rho}\right|_{T}^{(0)}(t-\tau)\right\} .
\end{aligned}
$$

Proof. In general, $-X \leqq x \leqq 2 X$ implies $\frac{x^{2}}{2}+\frac{n^{2} X^{2}}{2} \leqq(x+2 n X)^{2}$ except the case that $X<x \leqq 2 X$ and $n=-1$. We have for any $n$,

$$
\begin{aligned}
\mid D_{x}^{m} Z^{0}(x-\xi+2 n X, t ; \xi, \tau ; & \mu / \rho) \mid \leqq C_{2}^{(m)}(t-\tau)^{-\frac{1+m}{2}} \times \\
& \times \exp \left\{-(x-\xi)^{2} / 16\left|\frac{\mu}{\rho}\right|_{T}^{(0)}(t-\tau)\right\} \times \\
& \times \exp \left\{-X^{2} n^{2} / 16\left|\frac{\mu}{\rho}\right|_{T}^{(0)}(t-\tau)\right\},
\end{aligned}
$$

and for any $n(\neq-1), \quad\left|D_{x}^{m} Z^{0}(x+\xi+2 n X, t ; \xi, \tau ; \mu / \rho)\right|$ has the same bound. Hence we have

(2.14) $\sum_{n=-\infty}^{\infty}\left|D_{x}^{m} Z^{0}(x-\xi+2 n X, t ; \xi, \tau ; \mu / \rho)\right| \leqq C_{2}^{(m)}\left\{1+\frac{4\left(\left.\pi|\mu| \rho\right|_{T} ^{(0)} T\right)^{1 / 2}}{X}\right\} \times$

$$
\begin{aligned}
& \times(t-\tau)^{-\frac{1+m}{2}} \exp \left\{-(x-\xi)^{2} / 16\left|\frac{\mu}{\rho}\right|_{T}^{(0)}(t-\tau)\right\} \\
& \left(\sum_{n=-\infty}^{-1}+\sum_{n=0}^{\infty}\right)\left|D_{x}^{m} Z^{0}(x+\xi+2 n X, t ; \xi, \tau ; \mu / \rho)\right| \leqq \\
& \leqq C_{2}^{(m)}\left\{1+\frac{4\left(\pi|\mu / \rho|_{T}^{(0)} T\right)^{1 / 2}}{X}\right\}(t-\tau)^{-\frac{1+m}{2}} \times \\
& \quad \times \exp \left\{-(x-\xi)^{2} / 16\left|\frac{\mu}{\rho}\right|_{T}^{(0)}(t-\tau)\right\}
\end{aligned}
$$

In a direct way for $n=-1$, we obtain

$$
\begin{aligned}
\mid D_{x}^{m} Z^{0}(x+\xi-2 X, t & ; \xi, \tau ; \mu / \rho) \mid \leqq C_{2}^{(m)}(t-\tau)^{-\frac{1+m}{2} \times} \\
& \times \exp \left\{-(x-\xi)^{2} / 16\left|\frac{\mu}{\rho}\right|_{T}^{(0)}(t-\tau)\right\} .
\end{aligned}
$$


Thus, by (2.14), (2.15) and (2.16), we have finally

$$
\begin{aligned}
\left|D_{x}^{n} Z(x-\xi, t ; \xi, \tau ; \mu / \rho)\right| \leqq & C_{6}^{(m)}(t-\tau)^{-\frac{1+m}{2} \times} \\
& \times \exp \left\{-(x-\xi)^{2} / 16\left|\frac{\mu}{\rho}\right|_{T}^{(0)}(t-\tau)\right\},
\end{aligned}
$$

where $\quad C_{6}^{(m)}=C_{2}^{(m)}\left\{3+\frac{8\left(\pi|\mu / \rho|_{T}^{(0)} T\right)^{1 / 2}}{X}\right\}$.

Q.E.D.

Making use of the same procedure as in the proof of Lemma 2.4, we have

\section{Lemma 2.5.}

(i) For $t>t^{\prime}>\tau$,

$$
\begin{aligned}
&\left|D_{x}^{m} Z(x-\xi, t ; \xi, \tau ; \mu / \rho)-D_{x}^{m} Z\left(x-\xi, t^{\prime} ; \xi, \tau ; \mu / \rho\right)\right| \leqq C_{7}^{(m)}\left(t-t^{\prime}\right) \times \\
& \times\left(t^{\prime}-\tau\right)^{-\frac{2+m}{2}} \exp \left\{-(x-\xi)^{2} / 16\left|\frac{\mu}{\rho}\right|_{T}^{(0)}(t-\tau)\right\}
\end{aligned}
$$

(ii) $\quad\left|D_{x}^{m} Z(x-\xi, t ; \xi, \tau ; \mu / \rho)-D_{x^{\prime}}^{m} Z\left(x^{\prime}-\xi, t ; \xi, \tau ; \mu / \rho\right)\right| \leqq C_{8}^{(m)}\left|x-x^{\prime}\right| \times$

$$
\times(t-\tau)^{-\frac{2+m}{2}} \exp \left\{-(x-\xi)^{2} / 16\left|\frac{\mu}{\rho}\right|_{T}^{(0)}(t-\tau)\right\}
$$

(iii) $\quad\left|D_{t}^{k} D_{z}^{m} Z(z, t ; \xi, \tau ; \mu / \rho)-D_{t}^{k} D_{z}^{m} Z\left(z, t ; \xi^{\prime}, \tau ; \mu / \rho\right)\right| \leqq C_{9}^{(k, m)}\left|\xi-\xi^{\prime}\right|^{\alpha} \times$

$$
\times(t-\tau)^{-\frac{1+m+2 k}{2}} \exp \left\{-z^{2} / 16\left|\frac{\mu}{\rho}\right|_{T}^{(0)}(t-\tau)\right\} .
$$

Furthermore, by Lemma 2.5 , we have

\section{Lemma 2.6.}

$$
\left|\int_{0}^{X} D_{t}^{k} D_{x}^{m} Z(x-\xi, t ; \xi, \tau ; \mu / \rho) d \xi\right| \leqq C_{10}(t-\tau)^{-\frac{2 k+m-\alpha}{2}}, \text { for } 2 k+m>0
$$

It is easily seen that the fundamental solution $\Gamma(x, t ; \xi, \tau ; \mu / \rho)$ of

$$
\frac{\partial v}{\partial t}(x, t)=\frac{\mu}{\rho(x, t)} \frac{\partial^{2} v}{\partial x^{2}}(x, t) \quad(T \geqq t>0)
$$

with (2.2) is given in the form 


$$
\begin{aligned}
\Gamma(x, t ; \xi, \tau ; \mu / \rho)= & Z(x-\xi, t ; \xi, \tau ; \mu / \rho)+ \\
& +\int_{\tau}^{t} d \sigma \int_{0}^{x} K(x, t ; y, \sigma ; \mu / \rho) \Phi(y, \sigma ; \xi, \tau ; \mu / \rho) d y .
\end{aligned}
$$

The function $\Phi$ satisfies a Volterra-type integral equation:

$$
\begin{aligned}
\Phi(x, t ; \xi, \tau ; \mu / \rho)= & K(x, t ; \xi, \tau ; \mu / \rho)+ \\
& +\int_{\tau}^{t} d \sigma \int_{0}^{x} K(x, t ; y, \sigma ; \mu / \rho) \Phi(y, \sigma ; \xi, \tau ; \mu / \rho) d y,
\end{aligned}
$$

where

$$
K(x, t ; \xi, \tau ; \mu / \rho)=\left\{\frac{\mu}{\rho(x, t)}-\frac{\mu}{\rho(\xi, \tau)}\right\} D_{x}^{2} Z(x-\xi, t ; \xi, \tau ; \mu / \rho)
$$

The function $\Phi$ is given in the form

$$
\Phi(x, t ; \xi, \tau ; \mu / \rho)=\sum_{m=0}^{\infty} K_{m}(x, t ; \xi, \tau ; \mu / \rho),
$$

where

$$
\left\{\begin{array}{l}
K_{0}(x, t ; \xi, \tau ; \mu / \rho)=K(x, t ; \xi, \tau ; \mu / \rho) \\
K_{m}(x, t ; \xi, \tau ; \mu / \rho)=\int_{\tau}^{t} d \sigma \int_{0}^{x} K(x, t ; y, \sigma ; \mu / \rho) K_{m-1}(y, \sigma ; \xi, \tau ; \mu / \rho) d y
\end{array}\right.
$$

We shall prove the convergence of the series in (2.21) and estimate $\Gamma$ similarly to the way in which we $\operatorname{did} Z$ in Lemmas 2.5 and 2.6.

Lemma 2.7. $|K(x, t ; \xi, \tau ; \mu / \rho)| \leqq C_{11}(t-\tau)^{-\frac{3-\alpha}{2} \times}$

$$
\times \exp \left\{x(-\xi)^{2} / 32\left|\frac{\mu}{\rho}\right|_{T}^{(0)}(t-\tau)\right\}
$$

Proof. This follows directly from the Hölder continuity of $\frac{\mu}{\rho}$ and Lemma $2.4(m=2)$.

Q.E.D.

Proceeding similarly to evaluate $K_{1}, K_{2}$, etc., for any integer $m \geqq 0$ we have

Lemma 2.8 . 


$$
\begin{aligned}
\left|K_{m}\left(x, t ; \xi, \tau ; \frac{\mu}{\rho}\right)\right| & \leqq\left[\frac{\left\{2\left(2 \pi\left|\frac{\mu}{\rho}\right|_{T}^{(0)}\right)^{1 / 2} C_{11} \Gamma(\alpha / 2) T^{\alpha / 2}\right\}^{m+1}}{2\left(2 \pi\left|\frac{\mu}{\rho}\right|_{T}^{(0)}\right)^{1 / 2} \Gamma((m+1) \alpha / 2)} T^{-\alpha / 2}\right]_{I} \times \\
& \times(t-\tau)^{-\frac{3-\alpha}{2}} \exp \left\{-(x-\xi)^{2} / 32\left|\frac{\mu}{\rho}\right|_{T}^{(0)}(t-\tau)\right\} .
\end{aligned}
$$

From Lemma 2.8, it follows that the series expansion of $\Phi(x, t ; \xi, \tau$; $\left.\frac{\mu}{\rho}\right)$ is uniformly convergent for $T \geqq t \geqq 0$ and $\Phi$ is evaluated as follows:

Lemma 2.9. $|\Phi(x, t ; \xi, \tau ; \mu / \rho)| \leqq C_{12}(t-\tau)^{-\frac{3-\alpha}{2} \times}$

$$
\times \exp \left\{-(x-\xi)^{2} / 32\left|\frac{\mu}{\rho}\right|_{T}^{(0)}(t-\tau)\right\}
$$

where $C_{12}=\sum_{m=0}^{\infty}[\cdots]_{I}$ in Lemma 2.8 .

Thus, using Lemmas 2.4 and 2.9, we have

Lemma 2.10. $\left|D_{x}^{m} \Gamma(x, t ; \xi, \tau ; \mu / \rho)\right| \leqq C_{13}^{(m)}(t-\tau)^{-\frac{1+m}{2}} \times$

$$
\times \exp \left\{-(x-\xi)^{2} / 32\left|\frac{\mu}{\rho}\right|_{T}^{(0)}(t-\tau)\right\} .
$$

In order to study $\Gamma$ in more detail, we shall need the following lemmas.

Lemma 2.11. $\left|K(x, t ; \xi, \tau ; \mu / \rho)-K\left(x^{\prime}, t ; \xi, \tau ; \mu / \rho\right)\right| \leqq C_{14}(t-\tau)^{-3 / 2} \times$

$$
\times\left|x-x^{\prime}\right|^{\alpha} \exp \left\{-(x-\zeta)^{2} / 32\left|\frac{\mu}{\rho}\right|_{T}^{(0)}(t-\tau)\right\} .
$$

Proof. From (2.20), it follows that the lemma holds, by using the Hölder continuity of $\frac{\mu}{\rho}$, Lemmas 2.4 and 2.5 Q.E.D.

By induction, we obtain

Lemma 2.12. $\left|\Phi(x, t ; \xi, \tau ; \mu / \rho)-\Phi\left(x^{\prime}, t ; \xi, \tau ; \mu / \rho\right)\right| \leqq C_{15}(t-\tau \rho)^{-\frac{3}{2}} \times$

$$
\times\left|x-x^{\prime}\right|^{\alpha} \exp \left\{-(x-\xi)^{2} / 32\left|\frac{\mu}{\rho}\right|_{T}^{(0)}(t-\tau)\right\} .
$$

As a result, by using Lemmas $2.4,2.5,2.6,2.9$ and 2.12 , we have, after 
lengthy calculations,

\section{Lemma 2.13.}

(i ) $\left|\Gamma(x, t ; \xi, \tau ; \mu / \rho)-\Gamma\left(x, t^{\prime} ; \xi, \tau ; \mu / \rho\right)\right| \leqq C_{16}^{(0)}\left(t-t^{\prime}\right)\left(t^{\prime}-\tau\right)^{-\frac{3}{2}} \times$

$$
\times \exp \left\{-(x-\xi)^{2} / 32\left|\frac{\mu}{\rho}\right|_{T}^{(0)}(t-\tau)\right\}
$$

(ii) $\left|D_{x}^{m} \Gamma(x, t ; \xi, \tau ; \mu / \rho)-D_{x}^{m} \Gamma\left(x, t^{\prime} ; \xi, \tau ; \mu / \rho\right)\right| \leqq C_{16}^{(m)}\left\{\left(i-t^{\prime}\right)\left(t^{\prime}-\tau\right)^{-\frac{3}{2}}+\right.$

$$
\left.+\left(t-t^{\prime}\right)^{\frac{2-m+\alpha}{2}}\left(t^{\prime}-\tau\right)^{-\frac{3}{2}}\right\} \exp \left\{-(x-\zeta)^{2} / 32\left|\frac{\mu}{\rho}\right|_{T}^{(0)}(t-\tau)\right\} .
$$

\section{§3. The Existence of a Temporally Local Solution of}

$$
\text { (1.1), (2.1) and (2.2) }
$$

In the first place, we construct the sequence $\left\{v^{n}(x, t)\right\}$ such that

$$
\left\{\begin{array}{l}
v^{0}(x, t)=v_{0}(x) \in H_{T}^{2+\alpha} \\
v^{n}(x, t)=v_{0}(x)+\int_{0}^{t} d \tau \int_{0}^{x} \Gamma\left(x, t ; \xi, \tau ; \mu / \rho_{n-1}\right) N_{n-1}(\xi, \tau) d \xi
\end{array}\right.
$$

$(0 \leqq t \leqq T)$, where $\rho_{n-1}=\rho v_{n-1}$ and $N_{n-1}=\frac{\mu}{\rho_{n-1}} v_{0}^{\prime \prime}+v^{n-1} v_{x}^{n-1}$ (cf. (2.6)). We also assume (2.3) for $t=0$, i.e.,

$$
v_{0}^{\prime \prime}(x)_{\mid x=0}=v_{0}^{\prime \prime}(x)_{\mid x=X}=0 .
$$

The functions $v^{n}(n=1,2, \ldots)$ satisfy

$$
v_{t}^{n}=\frac{\mu}{\rho_{n-1}} v_{x x}^{n}-v^{n-1} v_{x}^{n-1}
$$

and

$$
v^{n}(x, 0)=v_{0}(x), \quad v^{n}(0, t)=v^{n}(X, t)=0 .
$$

By using the lemmas obtained in $\S 2$, especially Lemmas 2.10 and 2.13 , we have

\section{Lemma 3.1.}


( i ) $\left\|v^{n}\right\|_{T}^{(1)} \leqq C_{17,1}\left(T,\left\|\frac{\mu}{\rho_{n-1}}\right\|_{T}\right)\left|N_{n-1}\right|_{T}^{(0)}+\left\|v_{0}\right\|^{(1)}$

where $\left\|\frac{\mu}{\rho_{n-1}}\right\|_{T}=\left|\frac{\rho_{n-1}}{\mu}\right|_{T}^{(0)}+\left\|\frac{\mu}{\rho_{n-1}}\right\|_{T}^{(\alpha)}$,

(ii ) $\left|v_{x x}^{n}\right|_{T}^{(0)} \leqq C_{17,2}\left(T,\left\|\frac{\mu}{\rho_{n-1}}\right\|_{T}\right)\left\|N_{n-1}\right\|_{T}^{(\alpha)}+\left|v_{0}^{\prime \prime}\right|^{(0)}$,

(iii) $\quad\left|v^{n}\right|_{t, T}^{(\alpha / 2)} \leqq C_{17,3}\left(T,\left\|\frac{\mu}{\rho_{n-1}} \mid\right\|_{T}\right)\left|N_{n-1}\right|_{T}^{(0)}$,

(iv) $\quad\left|v_{x}^{n}\right|_{x, T}^{(\alpha)} \leqq 2\left(\left|v_{0}^{\prime \prime}\right|^{(0)}+\left|v_{0}^{\prime}\right|^{(0)}\right)+C_{17,4}\left(T,\left\|\frac{\mu}{\rho_{n-1}}\right\| \|_{T}\right)\left|N_{n-1}\right|^{(0)}$,

(v) $\quad\left|v_{x}^{n}\right|_{t, T}^{(\alpha / 2)} \leqq C_{17,5}\left(T,\left\|\frac{\mu}{\rho_{n-1}}\right\|_{T}\right)\left|N_{n-1}\right|_{T}^{(0)}$,

(vi) $\quad\left|v_{x x}^{n}\right|_{T}^{(\alpha)} \leqq C_{17,6}\left(T,\left\|\frac{\mu}{\rho_{n-1}}\right\| \|_{T}\right)\left\|N_{n-1}\right\|_{T}^{(\alpha)}+\left|v_{0}^{\prime \prime}\right|^{(\alpha)}$.

Remark. The constants $C_{17, i}(i=1,2,3,4,5)$ increase monotonically as each argument increases and $C_{17, i \downarrow 0}$ as $T \downarrow 0$.

It is easy to see that $v^{n-1} \in H_{T}^{2+\alpha}$ implies $\frac{\mu}{\rho_{n-1}} \in H_{T}^{\alpha}$ and $N_{n-1} \in H_{T}^{\alpha}$. Thus, by the above lemma, we see clearly that $v^{n} \in \hat{H}_{T}^{2+\alpha}$ and also $v^{n} \in$ $H_{T}^{2+\alpha}$. Hence by induction we obtain

Lemma 3.2. $v^{n}(x, t) \in H_{T}^{2+\alpha}$.

Now, we take an arbitrary constant $M_{0}$ such that

$$
\left\|v_{0}\right\|^{(2)}<M_{0}<+\infty \text {. }
$$

As for $\left\|\frac{\mu}{\rho_{n-1}}\right\| \|_{T}$, it holds that

$$
\begin{aligned}
& \left\|\frac{\mu}{\rho_{n-1}}\right\|_{T} \leqq \frac{\bar{\rho}_{0}}{\mu} \exp \left\{T\left|v_{x}^{n-1}\right|_{T}^{(0)}\right\}+\left(\bar{\rho}_{0}\right)^{-1} \exp \left\{T\left|v_{x}^{n-1}\right|_{T}^{(0)}\right\}+ \\
& \quad+2 \mu\left[\left\{\left(\bar{\rho}_{0}\right)^{-2}\left|\rho_{0}^{\prime}\right|^{(0)}+\left(\bar{\rho}_{0}\right)^{-1} T\left|v_{x x}^{n-1}\right|_{T}^{(0)} \exp \left\{2 T\left|v_{x}^{n-1}\right|_{T}^{(0)}\right\}\right\}+\right. \\
& \left.\quad+\left(\bar{\rho}_{0}\right)^{-1} \exp \left\{T\left|v_{x}^{n-1}\right|_{T}^{(0)}\right\}\right]+2 \mu\left[\left(\bar{\rho}_{0}\right)^{-1}\left(1+\left|v_{x}^{n-1}\right|_{T}^{(0)}\right) \times\right. \\
& \quad \times \exp \left\{T\left|v_{x}^{n-1}\right|_{T}^{(0)}\right\}+\left|v_{x}^{n-1}\right|_{T}^{(0)}\left\{\left(\bar{\rho}_{0}\right)^{-2}\left|\rho_{0}^{\prime}\right|^{(0)}+\right.
\end{aligned}
$$




$$
\left.\left.+\left(\bar{\rho}_{0}\right)^{-1} T\left|v_{x x}^{n-1}\right|_{T}^{(0)} \exp \left\{2 T\left|v_{x}^{n-1}\right|_{T}^{(0)}\right\}\right\}\right]
$$

If we assume that $\left\|v^{n-1}\right\|_{T}^{(2)}<M_{0}$, then we have

$$
\begin{aligned}
& \left\|\mid \frac{\mu}{\rho_{n-1}}\right\|_{T} \leqq\left\{\frac{\bar{\rho}_{0}}{\mu}+\mu\left(5+2 M_{0}\right)\left(\bar{\rho}_{0}\right)^{-1}\right\} \exp \left\{M_{0} T\right\}+ \\
& \quad+2 \mu\left(1+M_{0}\right)\left\{\left(\bar{\rho}_{0}\right)^{-2}\left|\rho_{0}^{\prime}\right|^{(0)}+\left(\bar{\rho}_{0}\right)^{-1} M_{0} T e^{2 M_{0} T}\right\} \equiv A\left(T, M_{0}\right) .
\end{aligned}
$$

By Lemma 3.1, we have $\left\|v^{n}\right\|_{T}^{(2)} \leqq\left\|v_{0}\right\|^{(2)}+\left(C_{17,1}+C_{17,2}\right)\left\|N_{n-1}\right\|_{T}^{(\alpha)}$. Furthermore, $\left\|v^{n-1}\right\|_{T}^{(2)}<M_{0}$ implies

$$
\left\|N_{n-1}\right\|_{T}^{(\alpha)} \leqq C_{18}\left(T, M_{0},\left\|\frac{\mu}{\rho_{n-1}}\right\|_{T}\right)
$$

where $C_{18}$ is monotonically increasing in each argument and $C_{18} \downarrow$ ' a certain positive constant' as $T \downarrow 0$. Therefore, we have

$$
\left\|v^{n}\right\|_{T}^{(2)} \leqq\left\|v_{0}\right\|_{T}^{(2)}+\left(C_{17,1}^{*}+C_{17,2}^{*}\right) C_{18}\left(T, M_{0}, A\left(T, M_{0}\right)\right),
$$

where $C_{17, i}^{*}=C_{17, i}\left(T, A\left(T, M_{0}\right)\right) \quad(i=1,2)$. Hence, for a sufficiently small $T_{1} \in(0, T]$

$$
\left\|v^{n}\right\|_{T_{1}}^{(2)} \leqq M_{0}
$$

By induction, for some $T_{2} \in(0, T]$

$$
\left\|v^{n}\right\|_{T_{2}}^{(2)} \leqq M_{0} \quad(n=1,2,3, \ldots),
$$

For simplicity we choose $T$ from the beginning in such a way that $T=T_{2}$.

In the next place, by (3.2) the differences $v^{n}-v^{n-1}$ satisfy the equation:

$$
\left(v^{n}-v^{n-1}\right)_{t}=\frac{\mu}{\rho_{n-1}}\left(v^{n}-v^{n-1}\right)_{x x}+\tilde{N}_{n-1}, \quad(n=1,2,3, \ldots),
$$

where $\tilde{N}_{n-1}=\left(\frac{\mu}{\rho_{n-1}}-\frac{\mu}{\rho_{n-2}}\right) v_{x x}^{n-1}+v^{n-1}\left(v^{n-1}-v^{n-2}\right)_{x}+\left(v^{n-1}-v^{n-2}\right) v_{x}^{n-2} \in$ $H_{T}^{\alpha}(n=2,3,4, \ldots)$, and by $(3.3)$ it also satisfies the initial-boundary conditions: 


$$
\left(v^{n}-v^{n-1}\right)(x, 0)=0, \quad\left(v^{n}-v^{n-1}\right)(0, t)=\left(v^{n}-v^{n-1}\right)(X, t)=0 .
$$

In the same way as we did in $\S 2$, we can construct the fundamental solution of (3.9) and (3.10), and the solution of (3.9) and (3.10) is uniquely expressed by

$$
\left(v^{n}-v^{n-1}\right)(x, t)=\int_{0}^{t} d \tau \int_{0}^{X} \Gamma\left(x, t ; \xi, \tau ; \mu / \rho_{n-1}\right) \tilde{N}_{n-1}(\xi, \tau) d \xi .
$$

Similarly to Lemma 3.1, we have the following lemma.

Lemma 3.3. $\left\|v^{n}-v^{n-1}\right\|_{T}^{(1)} \leq C_{19}\left(T,\left\|\frac{\mu}{\rho_{n-1}} \mid\right\|_{T}\right)\left|\tilde{N}_{n-1}\right|_{T}^{(0)}$ $(n=1,2,3, \ldots)$, where $C_{19}$ has the same property as $C_{17,1}$.

Directly by the above lemma, we have

$$
\left\|v^{n}-v^{n-1}\right\|_{T}^{(1)} \leqq C_{19}\left(T, A\left(T, M_{0}\right)\right)\left|\tilde{N}_{n-1}\right|_{T}^{(0)}
$$

\section{Lemma 3.4.}

$$
\left|\tilde{N}_{n-1}\right|_{T}^{(0)} \leqq C_{20}\left(T,\left|\left\|\frac{\mu}{\rho_{n-1}}\right\|_{T}+\left\|\frac{\mu}{\rho_{n-2}} \mid\right\|_{T}\right)\left\|v^{n-1}-v^{n-2}\right\|_{T}^{(1)}\right.
$$

where $C_{20}$ has the same property as $C_{18}$.

$$
\begin{array}{ll}
\text { Proof. } \quad & \left|\tilde{N}_{n-1}\right|_{T}^{(0)} \leqq\left|\frac{\mu}{\rho_{n-1}}-\frac{\mu}{\rho_{n-2}}\right|_{T}^{(0)}\left|v_{x x}^{n-1}\right|_{T}^{(0)}+ \\
& +\left|v^{n-1}\right|_{T}^{(0)}\left|\left(v^{n-1}-v^{n-2}\right)_{x}\right|_{T}^{(0)}+\left|v^{n-1}-v^{n-2}\right|_{T}^{(0)}\left|v_{x}^{n-2}\right|_{T}^{(0)} .
\end{array}
$$

Using Lemma 2.2, we have

$$
\left|\tilde{N}_{n-1}\right|_{T}^{(0)} \leqq C_{20}\left(T,\left\|\frac{\mu}{\rho_{n-1}}\right\|_{T}+\left\|\frac{\mu}{\rho_{n-2}}\right\|_{T}\right)\left\|v^{n-1}-v^{n-2}\right\|_{T}^{(1)}
$$

Q.E.D.

Hence we have

$$
\left|\tilde{N}_{n-1}\right|_{T}^{(0)} \leqq C_{20}\left(T, A\left(T, M_{0}\right)\right)\left\|v^{n-1}-v^{n-2}\right\|_{T}^{(1)} .
$$

Combining (3.12) and (3.13), we obtain 


$$
\left\|v^{n}-v^{n-1}\right\|_{T}^{(1)} \leq C_{21}\left\|v^{n-1}-v^{n-2}\right\|_{T}^{(1)},
$$

where $C_{21}$ has the same property as $C_{19}$.

By induction and (3.14), we have

$$
\left\|v^{n}-v_{n}^{n-1}\right\|_{T}^{(1)} \leqq C_{21}^{n-1}\left\|v^{1}-v^{0}\right\|_{T}^{(1)} .
$$

Since $C_{21} \downarrow 0$ as $T \downarrow 0$, it holds, for some $T_{0} \in(0, T]$, that $C_{21}\left(T_{0}, A\left(T_{0}\right.\right.$, $\left.\left(M_{0}\right)\right)<1$, whereas by Lemma 3.1

$$
\left\|v^{1}-v^{0}\right\|_{T_{0}}^{(1)} \leqq C_{17,1}\left(T_{0}, A\left(T_{0}, M_{0}\right)\right)\left|N_{0}\right|_{T_{0}}^{(0)}
$$

and

$$
\left|N_{0}\right|_{T_{0}}^{(0)} \leqq\left(\bar{\rho}_{0}\right)^{-1} M_{0} \exp \left\{M_{0} T_{0}\right\}+M_{0}^{2}<+\infty
$$

Thus

$$
\sum_{n=1}^{\infty} C_{21}^{n-1}\left\|v^{1}-v^{0}\right\|_{T_{0}}^{(1)}<+\infty
$$

Therefore, $\left\{v^{n}\right\}$ converges to an element $v$ of $H_{T}^{2+\alpha}$ as $n \rightarrow \infty$. As is known the expression (2.5), $\left\{\rho_{n}\right\}$ converges to an element $\rho_{v}$ of $B_{T}^{1}$ as $n \rightarrow \infty$. $\quad N_{n}$ also converges to $N=\frac{\mu}{\rho_{v}} v_{0}^{\prime \prime}+v v_{x}$. Hence, by the formula (2.16), (2.18), (2.25), (2.26), (2.27), (2.28) and (2.29), $Z^{0}(x-\xi, t ; \xi, \tau$; $\left.\mu / \rho_{n-1}\right), Z\left(x-\xi, t ; \xi, \tau ; \mu / \rho_{n-1}\right), K_{m}\left(x, t ; \xi, \tau ; \mu / \rho_{n-1}\right), \Phi\left(x, t ; \xi, \tau ; \mu / \rho_{n-1}\right)$, and $\Gamma\left(x, t ; \xi, \tau ; \mu / \rho_{n-1}\right)$ converge to $Z^{0}\left(x-\xi, t ; \xi, \tau ; \mu / \rho_{v}\right), \quad Z(x-\xi$, $\left.t ; \xi, \tau ; \mu / \rho_{v}\right), K_{m}\left(x, t ; \xi, \tau ; \mu / \rho_{v}\right), \Phi\left(x, t ; \xi, \tau ; \mu / \rho_{v}\right)$ and $\Gamma\left(x, t ; \xi, \tau ; \mu / \rho_{v}\right)$, respectively, as $n \rightarrow \infty$. Thus by (3.1), it holds, for $0 \leqq t \leqq T$, that

$$
\begin{gathered}
v(x, t)=v_{0}(x)+\int_{0}^{t} d \tau \int_{0}^{x} \Gamma\left(x, t ; \xi, \tau ; \mu / \rho_{v}\right)\left\{\frac{\mu}{\rho_{v}} v_{0}^{\prime \prime}(\xi)-\right. \\
\left.-v v_{\xi}(\xi, \tau)\right\} d \xi
\end{gathered}
$$

As a result, we have

Theorem 3.1. For some $T \in(0, \infty)$, there exists a solution of (1.1), (2.1) and (2.2) in $H_{T}^{2+\alpha} \times B_{T}^{1}$.

Remark. For $v$, Lemma 3.1 also holds. 


\section{§4. The Uniqueness of the Solution of (1.1), (2.1) and (2.2)} in $\boldsymbol{H}_{T}^{++\alpha} \times \boldsymbol{B}_{T}^{1}$.

Now let us direct ourselves towards the problem of uniqueness concerning the system (1.1) of differential equations. (cf. [11]). We assume that there exist two solutions $\left(v, \rho_{v}\right)$ and $\left(w, \rho_{w}\right)$ of (1.1) in $H_{T}^{2+\alpha} \times B_{T}^{1}$ satisfying one and the same initial-boundary conditions (2.1) and (2.2). The difference $v-w$ satisfies the equation (3.9) and the initialboundary condition (3.10) as $v^{n}$ and $v^{n-1}$ are replaced by $v$ and $w$ respectively. Then $v-w$ can be uniquely expressed in the form (3.11) as $v^{n}$ and $v^{n-1}$ are replaced by $v$ and $w$ respectively, i.e.,

$$
(v-w)(x, t)=\int_{0}^{t} d \tau \int_{0}^{x} \Gamma\left(x, t ; \xi, \tau ; \mu / \rho_{v}\right) \tilde{N}(\xi, \tau) d \xi,
$$

where $\tilde{N}(x, t)=\left(\frac{\mu}{\rho_{v}}-\frac{\mu}{\rho_{w}}\right)(x, t)-v(x, t)(v-w)_{x}(x, t)+(v-w)(x, t) w_{x}(x, t)$. As for $v-w$, in a way analogous to that used in the preceding section for $v^{n}-v^{n-1}$, we obtain

\section{Lemma 4.1.}

$$
\begin{aligned}
& \left\|v-w^{\cdot}\right\|_{T_{0}}^{(1)} \leqq C_{22}\left(T_{0},\left\|\frac{\mu}{\rho_{v}}\right\|_{T}+\left\|\frac{\mu}{\rho_{w}}\right\| \|_{T}\right)|\tilde{N}|_{T_{0}}^{(0)}, \\
& |\tilde{N}|_{T_{0}}^{(0)} \leqq C_{23}\left(T_{0},\left\|\frac{\mu}{\rho_{v}}\right\|_{T}+\left|\left\|\frac{\mu}{\rho_{w}} \mid\right\|_{T}\right)\left\|v-w^{\prime}\right\|_{T_{0}}^{(1)},\right.
\end{aligned}
$$

$\left(0<T_{0} \leqq T\right)$, where $C_{22}$ and $C_{23}$ have the same property as $C_{19}$ and $C_{20}$, respectively.

Finally, we have an inequality similar to (3.14):

$$
\|v-w\|_{T_{0}}^{(1)} \leqq C_{24}\left(T_{0} ; v, w\right)\|v-w\|_{T_{0}}^{(1)},
$$

where $C_{24}\left(T_{0} ; \cdots\right)$ has the same property as $C_{22}$.

Since $C_{24}\left(T_{0} ; \cdots\right) \downarrow 0$ as $T_{0} \downarrow 0$, it holds for a sufficiently small $T_{1} \in$ $(0, T]$, that 


$$
0 \leqq\left(1-C_{24}\left(T_{1}\right)\right)\|v-w\|_{T_{1}}^{(1)} \leqq 0
$$

Hence, we obtain $\|v-w\|_{T_{1}}^{(1)}=0$, i.e., $v(x, t)=w(x, t)\left(0 \leqq t \leqq T_{1} \leqq T\right)$. According to the assumption, we can continue this procedure again by starting at $t=T_{1}$. After a finite number of repetitions of this procedure, it is shown in a conventional way that the following assertion holds.

Theorem 4.1. If $(v, \rho)$ and $\left(w, \rho^{*}\right) \in H_{T}^{2+\alpha} \times B_{T}^{1} \quad$ satisfy $\quad(1.1)$, (2.1) and (2.2), then $(v, \rho)=\left(w, \rho^{*}\right)\left(\rho=\rho^{*}=\rho_{v}\right)$.

\section{§5. An a priori Estimate for $\left.|\rho|\right|_{T} ^{(0)}$}

We begin with the following well known lemmas. (see, e.g., [5], [15]).

Lemma 5.1. If $u(x, t)$ satisfies regularly the equation:

$$
\frac{\partial u}{\partial t}=a(x, t) \frac{\partial^{2} u}{\partial x^{2}}+b(x, t) \frac{\partial u}{\partial x}+c(x, t) u, \quad(0<t \leqq T)
$$

where $a(x, t), b(x, t)$ and $c(x, t)$ are continuous in $\bar{Q}_{T}$ and satisfy

$$
0 \leqq a(x, t) \leqq|a|_{T}^{(0)}<+\infty, \quad c(x, t) \leqq 0,
$$

then it holds that

$$
\max _{\bar{Q}_{T}}|u| \leqq \max _{\Gamma_{T}}|u|
$$

Lemma 5.2. If $u(x, t)$ satisfies regularly the equation:

$$
\frac{\partial u}{\partial t}=a(x, t) \frac{\partial^{2} u}{\partial x^{2}}+b(x, t) \frac{\partial u}{\partial x}+c(x, t) u+f(x, t),
$$

where $a(x, t)>0$ in $\bar{Q}_{T}$ and if

$$
D_{t}^{k} D_{x}^{m} a, D_{t}^{k} D_{x}^{m} b, D_{t}^{k} D_{x}^{m} c, D_{t}^{k} D_{x}^{m} f \quad(0 \leqq \mathrm{~m}+2 k \leqq p, k \leqq q)
$$

belong to $H_{T}^{\alpha}$, then $D_{t}^{k} D_{x}^{m} u(0 \leqq m+2 k \leqq p+2, k \leqq q+1)$ exist and are Hölder continuous (exponent $\alpha$ ) in $\bar{\Omega} \times\left[T^{\prime}, T\right]$ for an arbitrary $T^{\prime} \in$ $(0, T)$. 
Directly by the above lemmas, we have

Lemma 5.3. If $(v, \rho) \in H_{T}^{2+\alpha} \times B_{T}^{1} \quad$ satisfies (1.1), (2.1) and (2.2), then it holds that

$$
|v|_{i}^{(0)} \leqq\left|v_{0}\right|^{(0)}
$$

Lemma 5.4. If $(v, \rho) \in H_{T}^{2+\alpha} \times B_{T}^{1} \quad$ satisfies (1.1), (2.1), (2.2) and an additional condition:

$$
\rho_{0} \in H^{1+\alpha}
$$

then $v \in H_{\left[T^{\prime}, T\right]}^{3+\alpha}$ where the suffix $\left[T^{\prime}, T\right]$ denotes that $\bar{Q}_{T}$ in (1.4) and $(1.4)^{\prime}$ is replaced by $\bar{\Omega} \times\left[T^{\prime}, T\right]$. [We note that (5.5) implies $\rho \in B_{T}^{1+\alpha}$.]

Lemma 5.5. If $(v, \rho) \in H_{T}^{2+\alpha} \times B_{T}^{1} \quad$ satisfies (1.1), (2.1), (2.2) and (5.5), then it holds that

$$
\bar{\rho}_{0} \exp \left\{-\frac{1}{\mu}\left|\rho_{0} v_{0}\right|^{(0)} X\right\} \leqq \rho(x, t) \leqq \bar{\rho}_{0} \exp \left\{\frac{1}{\mu}\left|\rho_{0} v_{0}\right|^{(0)} X\right\}
$$

Proof. By (2.5), we need to estimate $\int_{0}^{x} \bar{v}_{x}(\tau ; x, t) d \tau$. Since $\frac{\partial}{\partial x} \int_{0}^{t} \bar{v}_{x}(\tau ; x, t) d \tau=\int_{0}^{t} \bar{v}_{x x}(\tau ; x, t) x_{x}(\tau ; x, t) d \tau=$

$$
\begin{gathered}
=\frac{\rho(x, t)}{\mu}\left\{v(x, t)-v_{0}\left(x_{0}(x, t)\right)\right\} \\
\int_{0}^{t} \bar{v}_{x}(\tau ; x, t) d \tau= \\
=\left\{\frac{1}{\mu} \int_{0}^{x} \rho v d x+\int_{0}^{t} \bar{v}_{x}(\tau ; 0, t) d \tau\right\}- \\
-\frac{1}{\mu} \int_{0}^{x} \rho(x, t) v_{0}\left(x_{0}(x, t)\right) d x
\end{gathered}
$$

The second term of the right-hand side of (5.7) is transformed, by using (2.6), as follows:

$$
\int_{0}^{x} \rho(x, t) v_{0}\left(x_{0}\right) d x=\int_{0}^{x_{0}} \rho_{0}\left(x_{0}^{\prime}\right) v_{0}\left(x_{0}^{\prime}\right) d x_{0}^{\prime}
$$

We denote the first term of the right-hand side of (5.7) by $\psi(x, t)$. Then, we have 


$$
\psi_{t}(x, t)=\frac{1}{\mu} \int_{0}^{x}\left(\rho_{t} v+\rho v_{t}\right) d x+v_{x}(0, t)=\frac{1}{\mu}\left(-\rho v^{2}+\mu v_{x}\right) .
$$

On the other hand, $\psi_{x}(x, t)=\frac{1}{\mu} \rho v,\left(\frac{\mu}{\rho} \psi_{x}\right)_{x}=v_{x}$. Hence, we have

$$
\begin{aligned}
& \psi_{t}=\frac{\mu}{\rho} \psi_{x x}-\left(\frac{\mu \rho_{x}}{\rho^{2}}+v\right) \psi_{x}, \\
& \psi(x, 0)=\frac{1}{\mu} \int_{0}^{x} \rho_{0} v_{0} d x, \psi_{x}(0, t)=\psi_{x}(X, t)=0 \quad(T \geqq t \geqq 0) .
\end{aligned}
$$

Therefore, $\psi(x, t)$ is to be expressed by utilizing the fundamental solution of the linear parabolic equation (5.8) in the following way:

$$
\psi(x, t)=\int_{0}^{x} \tilde{\Gamma}(x, t ; \xi, 0) \psi(\xi, 0) d \xi=\frac{1}{\mu} \int_{0}^{x} \tilde{\Gamma}(x, t ; \xi, 0)\left(\int_{0}^{\xi} \rho_{0} v_{0} d y\right) d \xi
$$

where $\tilde{\Gamma}$ is the fundamental solution of (5.8).

Hence, we have $\left|\psi-\frac{1}{\mu} \int_{0}^{x} \rho(x, t) v_{0}\left(x_{0}(x, t)\right) d x\right|_{T}^{(0)} \leqq \frac{1}{\mu}\left|\rho_{0} v_{0}\right|^{(0)} X$.

As a result, we obtain (5.6).

Q.E.D.

If $(v, \rho) \in H_{T}^{2+\alpha} \times B_{T}^{1}$ satisfies (1.1), (2.1), (2.2), and (5.5), then by Lemmas 2.1 and 5.2, we have

$$
\begin{aligned}
\left\|\frac{\mu}{\rho}\right\|_{T}^{(\alpha)} & \leqq 5\left|\frac{\mu}{\rho}\right|_{T}^{(0)}+2\left|\left(\frac{\mu}{\rho}\right)_{x}\right|_{T}^{(0)}+2\left|\left(\frac{\mu}{\rho}\right)_{t}\right|_{T}^{(0)} \\
& \leqq\left(5+2\left|v_{x}\right|_{T}^{(0)}\right) \mu\left(\bar{\rho}_{0}\right)^{-1} \exp \left\{\left|\int_{0}^{t} \bar{v}_{x}(\tau ; x, t) d \tau\right|_{T}^{(0)}\right\}+ \\
& +4\left|v_{0}\right|^{(0)}\left(1+\left|v_{0}\right|^{(0)}\right)+2 \mu\left(\bar{\rho}_{0}\right)^{-2}\left|\rho_{0}^{\prime}\right|^{(0)}\left(1+\left|v_{0}\right|^{(0)}\right),
\end{aligned}
$$

since

$$
\left\{\begin{array}{l}
\left(\frac{\mu}{\rho}\right)_{x}=-\frac{\mu \rho_{x}}{\rho^{2}}=-\frac{\rho_{0}^{\prime}}{\rho_{0}^{2}}+v(x, t)-v_{0}\left(x_{0}(x, t)\right), \\
\left(\frac{\mu}{\rho}\right)_{t}=-\frac{\mu \rho_{t}}{\rho^{2}}=\mu\left\{\frac{v_{x}}{\rho}-v\left(\frac{1}{\rho}\right)_{x}\right\} .
\end{array}\right.
$$

By (5.11), we know that, in order to have an a priori estimate for $\left\|\frac{\mu}{\rho}\right\|_{T}^{(\alpha)}$, we have to obtain beforehand one for $\left|v_{x}\right|_{T}^{(0)}$. Hereafter in $\S 6$, we shall endeavor to have an a priori estimate for $\left|v_{x}\right|_{T}^{(0)}$. 


\section{§6. An a priori Estimate for $\left|\boldsymbol{v}_{\boldsymbol{x}}\right|_{\mathrm{T}}^{(0)}$}

Lemma 6.1. Under the initial-boundary conditions (2.1), (2.2) and (5.5), $\left|v_{x}\right|_{T}^{(0)}$ is bounded by a constant depending only on the quantities appearing in (2.1), (2.2) and (5.5) but independent of $T$.

Proof. The procedure of the demonstration is divided into three steps.

((1-st step)). First of all, we note that (5.6) holds by Lemma 5.5. Now we define $v_{\lambda}(x, t)$ by

$$
v_{\lambda}(x, t) \equiv v_{x}(x, t)^{2}+\lambda v(x, t)^{2},
$$

where $\lambda$ is a constant to be determined later.

Since $v \in H_{\left[T^{\prime}, T\right]}^{3+\alpha}$ by Lemma 5.4, $v_{x}$ satisfies the equation:

$$
\left(v_{x}\right)_{t}=\frac{\mu}{\rho}\left(v_{x}\right)_{x x}+\left\{\left(\frac{\mu}{\rho}\right)_{x}-v\right\}\left(v_{x}\right)_{x}-\left(v_{x}\right)^{2} .
$$

Let $\mathscr{L}$ be defined by

$$
\mathscr{L} \equiv \frac{\partial}{\partial t}-\frac{\mu}{\rho} \frac{\partial^{2}}{\partial x^{2}}+v \frac{\partial}{\partial x}
$$

Then, we have for any $\varepsilon>0$

$$
\begin{aligned}
\mathscr{L} v_{\lambda}= & 2\left(\frac{\mu}{\rho}\right)_{x} v_{x} v_{x x}-2 v_{x}^{3}-2 \frac{\mu}{\rho} v_{x x}^{2}-2 \frac{\mu \lambda}{\rho} v_{x}^{2} \\
\leqq & {\left[2\left\{\left(\bar{\rho}_{0}\right)^{-2}\left|\rho_{0}^{\prime}\right|^{(0)}+2\left|v_{0}\right|^{(0)}\right\} \varepsilon-\frac{2 \mu}{|\rho|_{T}^{(0)}}\right] v_{x x}^{2}+} \\
& +\left[\frac{1}{2 \varepsilon}\left\{\left(\bar{\rho}_{0}\right)^{-2}\left|\rho_{0}^{\prime}\right|^{(0)}+2\left|v_{0}\right|^{(0)}\right\}+2\left|v_{x}\right|_{T}^{(0)}-\right. \\
& \left.-\frac{2 \mu \lambda}{|\rho|_{T}^{(0)}}\right] v_{x}^{2} .
\end{aligned}
$$

We choose $\varepsilon=\varepsilon_{0}$ in such a way that 


$$
\varepsilon_{0} \equiv\left\{\begin{array}{l}
1 \quad\left(\text { if }\left|\rho_{0}^{\prime}\right|^{(0)}=\left|v_{0}\right|^{(0)}=0\right) \\
\frac{\mu}{|\rho|_{T}^{(0)}}\left\{\left(\bar{\rho}_{0}\right)^{-2}\left|\rho_{0}^{\prime}\right|^{(0)}+2\left|v_{0}\right|^{(0)}\right\}^{-1}
\end{array}\right.
$$

(otherwise).

For such a fixed number $\varepsilon_{0}(>0)$, it holds that

$$
\begin{aligned}
\mathscr{L} v_{\lambda} \leqq & {\left[\frac{1}{2 \varepsilon_{0}}\left\{\left(\bar{\rho}_{0}\right)^{-2}\left|\rho_{0}^{\prime}\right|^{(0)}+2\left|v_{0}\right|^{(0)}\right\}+2\left|v_{x}\right|_{T}^{(0)}-\right.} \\
& \left.-\frac{2 \mu \lambda}{|\rho|_{T}^{(0)}}\right] v_{x}^{2} .
\end{aligned}
$$

If we take $\lambda=\lambda_{0} \equiv \frac{|\rho|_{T}^{(0)}}{2 \mu}\left[\frac{1}{2 \varepsilon_{0}}\left\{\left(\bar{\rho}_{0}\right)^{-2}\left|\rho_{0}^{\prime}\right|^{(0)}+2\left|v_{0}\right|^{(0)}\right\}+2\left|v_{x}\right|_{T}^{(0)}\right]$, then we have an inequality

$$
\mathscr{L} v_{\lambda_{0}} \leqq 0
$$

By (6.7) and the maximum principle, it holds that

$$
\begin{aligned}
\max _{\overline{Q_{T}}} v_{\lambda_{0}} & \leqq \max _{\Gamma_{T}}\left(v_{x}^{2}+\lambda_{0} v^{2}\right) \\
& \leqq\left(\left|v_{0}^{\prime}\right|^{(0)}\right)^{2}+\lambda_{0}\left(\left|v_{0}\right|^{(0)}\right)^{2}+\max _{S_{T}} v_{x}^{2} .
\end{aligned}
$$

((2-nd step)). To evaluate the last term of (6.8), it is clear the case $\left|v_{0}\right|^{(0)}=0$. Then, suppose $\left|v_{0}\right|^{(0)} \neq 0$ and consider $v=\phi(w)$, where $\phi$ is a smooth function to be determined later. Thus, we get

$$
\mathscr{L} v=\phi^{\prime}\left[w_{t}-\frac{\mu}{\rho} w_{x x}-\frac{\mu}{\rho} \frac{\phi^{\prime \prime}}{\phi^{\prime}} w_{x}^{2}+\frac{1}{\phi^{\prime}} v v_{x}\right]=0 \text {. }
$$

If $\phi^{\prime}>0$ and $\phi^{\prime \prime}<0$, then it follows that

$$
\begin{aligned}
w_{t}-\frac{\mu}{\rho} w_{x x}= & \frac{\phi^{\prime \prime}}{\phi^{\prime}} \frac{\mu}{\rho} w_{x}^{2}-\frac{1}{\phi^{\prime}} v v_{x} \\
\leqq & \frac{\mu}{|\rho|_{T}^{(0)}}\left\{\left[\frac{\phi^{\prime \prime}}{\phi^{\prime}}+\frac{|\rho|_{T}^{(0)}\left|v_{0}\right|^{(0)}}{2 \mu} \phi^{\prime}\right] w_{x}^{2}+\right. \\
& \left.+\frac{|\rho|_{T}^{(0)}\left|v_{0}\right|^{(0)}}{2 \mu} \frac{1}{\phi^{\prime}}\right\}
\end{aligned}
$$


Furthermore, we choose $\phi$ in such a way that

$$
\frac{\phi^{\prime \prime}}{\phi^{\prime}}+\frac{|\rho|_{T}^{(0)}\left|v_{0}\right|^{(0)}}{2 \mu} \phi^{\prime} \leqq 0 \text { and } \phi(0)=0
$$

that is to say, for example,

$$
\begin{aligned}
& \phi(w)=\frac{2 \mu}{|\rho|_{T}^{(0)}\left|v_{0}\right|^{(0)}} \log (1+w), \\
& w=-1+\exp \left\{\frac{2 \mu}{|\rho|_{T}^{(0)}\left|v_{0}\right|^{(0)}} v\right\} .
\end{aligned}
$$

By (6.11), it is clear that $w_{!} s_{T}=0$.

For such a function $\phi$, it follows from (6.10) that

$$
w_{t}-\frac{\mu}{\rho} w_{x x} \leqq \frac{|\rho|_{T}^{(0)}\left(\left|v_{0}\right|^{(0)}\right)^{2}}{4 \mu}-\exp \left\{\frac{2 \mu}{|\rho|_{T}^{(0)}}\right\} \equiv C_{25} .
$$

Differentiating both sides of (6.11) once in $x$ and putting $t=0$, we get

$$
\max _{\bar{\Omega}}\left|w_{x}(x, 0)\right| \leqq \frac{2 \mu}{|\rho|_{T}^{(0)}\left|v_{0}\right|^{(0)}}\left|v_{0}^{\prime}\right|^{(0)} \exp \left\{\frac{2 \mu}{|\rho|_{T}^{(0)}}\right\} .
$$

Define the constant $C_{26}$ by $\max \left\{\frac{2 \mu\left|v_{0}^{\prime}\right|^{(0)}}{|\rho|_{T}^{(0)}\left|v_{0}\right|^{(0)}} \exp \left\{\frac{2 \mu}{|\rho|_{T}^{(0)}}\right\}, 1\right\}$, then it follows that

$$
\max _{\bar{\Omega}}\left|w_{x}(x, 0)\right| \leqq C_{26}
$$

Now, consider the function $w(x, t)+v e^{-x}$. For $v \geqq C_{26} e^{x}, w(x, t)+v e^{-x} \geqq 0$ and

$$
\max _{\Gamma_{T}}\left\{w+v e^{-x}\right\} \leqq \max _{S_{T}^{0}}\left\{w+v e^{-x}\right\}=v
$$

since $\frac{\partial}{\partial x}\left\{w(x, 0)+v e^{-x}\right\}_{\mid x \in \bar{\Omega}} \leqq C_{26}-v e^{-X} \leqq 0$.

Next, if we take $v=v_{0} \equiv e^{X} \max \left\{C_{26}, \frac{|\rho|_{T}^{(0)}}{\mu} C_{25}\right\}$, then

$$
\frac{\partial}{\partial t}\left\{w+v_{0} e^{-x}\right\}-\frac{\mu}{\rho} \frac{\partial^{2}}{\partial x^{2}}\left\{w+v_{0} e^{-x}\right\} \leqq 0,
$$


because it holds that

$$
w_{t}-\frac{\mu}{\rho}\left\{w+v_{0} e^{-x}\right\}_{x x} \leqq C_{25}-\frac{\mu v_{0}}{|\rho|_{T}^{(0)}} e^{-X} \leqq 0
$$

Hence, by (6.15) and (6.16), the maximum of $w+v_{0} e^{-x}$ in $\bar{Q}_{T}$ is attained at all points of $S_{T}^{0}$. Thus

$$
\left.\frac{\partial w}{\partial x}\right|_{s_{T}^{\circ} \leqq v_{0}}
$$

Directly, from (6.17), we have

$$
\left.\frac{\partial v}{\partial x}\right|_{s_{T}^{0}} \leqq \frac{2 \mu v_{0}}{|\rho|_{T}^{(0)}\left|v_{0}\right|^{(0)}}
$$

In order to obtain the estimate for $\left.\frac{\partial v}{\partial x}\right|_{s_{T}^{0}}$ from below, it is sufficient to apply the above one to the solution $-v(x, t)$ of the equation:

$$
(-v)_{t}-\frac{\mu}{\rho}(-v)_{x x}-v v_{x}=0 .
$$

As a result, we have

$$
\left|\frac{\partial v}{\partial x}\right|_{s_{T}^{0}} \mid \leqq \frac{2 \mu v_{0}}{|\rho|_{T}^{(0)}\left|v_{0}\right|^{(0)}}
$$

As for $\left.\frac{\partial v}{\partial x}\right|_{s_{T}^{x}}$, consider the function $\tilde{v}(x, t)=v(X-x, t)$, and repeat the same argument for $\tilde{v}_{x}$ on $S_{T}^{0}$ as for $v_{x}$ on $S_{T}^{0}$. Finally we get

$$
\max _{\boldsymbol{S}_{T}}\left|v_{x}\right| \leqq \frac{2 \mu v_{0}}{|\rho|_{T}^{(0)}\left|v_{0}\right|^{(0)}} .
$$

((3-rd step)). By (6.8) and (6.20), we have

$$
\begin{aligned}
\left(\left|v_{x}\right|_{T}^{(0)}\right)^{2} \leqq & \left(\left|v_{0}^{\prime}\right|^{(0)}\right)^{2}+\lambda_{0}\left(\left|v_{0}\right|^{(0)}\right)^{2}+ \\
& +\left(\frac{2 \mu v_{0}}{|\rho|_{T}^{(0)}\left|v_{0}\right|^{(0)}}\right)^{2} \\
& \leqq\left(\left|v_{0}^{\prime}\right|^{(0)}\right)^{2}+\left(\left|v_{0}\right|^{(0)}\right)^{2} \frac{|\rho|_{T}^{(0)}}{2 \mu}\left[\frac { 1 } { 2 \varepsilon _ { 0 } } \left\{\left(\bar{\rho}_{0}\right)^{-2}\left|\rho_{0}^{\prime}\right|^{(0)}+\right.\right.
\end{aligned}
$$




$$
\begin{aligned}
& \left.\left.+2\left|v_{0}\right|^{(0)}\right\}+2\left|v_{x}\right|_{T}^{(0)}\right]+\left(\frac{2 \mu v_{0}}{|\rho|_{T}^{(0)}\left|v_{0}\right|^{(0)}}\right)^{2} \\
\equiv & a_{0}+b_{0}\left|v_{x}\right|_{T}^{(0)} .
\end{aligned}
$$

where

$$
\left\{\begin{aligned}
a_{0}=\left(\left|v_{0}^{\prime}\right|^{(0)}\right)^{2}+ & \frac{|\rho|_{T}^{(0)}}{2 \mu} \frac{1}{2 \varepsilon_{0}}\left\{\left(\bar{\rho}_{0}\right)^{-2}\left|\rho_{0}^{\prime}\right|^{(0)}+2\left|v_{0}\right|^{(0)}\right\} \times \\
& \times\left(\left|v_{0}\right|^{(0)}\right)^{2}+\left(\frac{2 \mu v_{0}}{|\rho|_{T}^{(0)}\left|v_{0}\right|^{(0)}}\right)^{2} \\
b_{0}= & \frac{|\rho|_{T}^{(0)}\left(\left|v_{0}\right|^{(0)}\right)^{2}}{\mu}
\end{aligned}\right.
$$

Thus, it holds that

$$
\left|v_{x}\right|_{T}^{(0)} \leqq \frac{b_{0}+\left(b_{0}^{2}+4 a_{0}\right)^{1 / 2}}{2}
$$

From (5.11), it follows that

$$
\left\|\frac{\mu}{\rho}\right\|_{T}^{(\alpha)} \leqq C_{27}\left(v_{0}, \rho_{0}\right)
$$

§7. An a priori Estimate for \|\|$_{r}^{(2+\alpha)}$ and the Main Theorem

By Lemma 3.1 for $v$ instead of $v^{n}$, we have

$$
\text { Lemma 7.1. } \quad\|v\|_{\Gamma}^{(1+\alpha)} \leqq C_{28}\left(T ; v_{0}, \rho_{0}\right),
$$

where $C_{28}(T ; \cdots)$ increases monotonically as $T$ increases.

Hence, from this, it follows that

$$
\|N\|_{T}^{(\alpha)} \leqq C_{29}\left(T ; v_{0}, \rho_{0}\right)
$$

where $C_{29}(T ; \cdots)$ has the same property as $C_{28}$. Thus, we have

$$
\text { Lemma 7.2. } \quad\left\|v_{x x}\right\|_{T}^{(\alpha)} \leqq C_{30}\left(T ; v_{0}, \rho_{0}\right) .
$$

From the discussions made thus far follows:

Lemma 7.3. Under the initial-boundary conditions (2.1), (2.2) 
and (5.5), if there exists a solution $(v, \rho) \in H_{T}^{2+\alpha} \times B_{T}^{1}$ of (1.1), then $\|v\|_{T}^{(2+\alpha)}+[\rho]_{T}^{(1)}$ has a priori bounds in $T$, where

$$
[\rho]_{T}^{(n)} \equiv \sum_{r+s=0}^{n}\left|D_{t}^{r} D_{x}^{s} \rho\right|_{T}^{(0)} \quad(r \text { and } s, \text { integer } s \geqq 0) .
$$

Proof. We have only to note that

$$
\begin{aligned}
\|v\|_{T}^{(2+\alpha)} \leqq & v\left\|_{T}^{(1+\alpha)}+\left(1+\left\|\frac{\mu}{\rho}\right\|_{T}^{(\alpha)}\right)\right\| v_{x x} \|_{T}^{(\alpha)}+ \\
& +\left\|v v_{x}\right\|_{T}^{(\alpha)} .
\end{aligned}
$$

The results obtained since $\$ 5$ guarantee that each term of the righthand side of the above inequality has $a$ priori bounds in $T$.

Q.E.D.

Combining Theorems 3.1, 4.1 and Lemma 7.3, we have the following main theorem on the existence of a temporally global solution of (1.1), (2.1), (2.2) and (5.5).

Theorem 7.1. Under the initial-boundary conditions (2.1), (2.2) and (5.5), there uniquely exists a regular temporally global solution and it holds that

$$
\left\{\begin{array}{l}
|v(x, t)| \leqq\left|v_{0}\right|^{(0)}, \\
0 \leqq \bar{\rho}_{0} \exp \left\{-\frac{1}{\mu}\left|\rho_{0} v_{0}\right|^{(0)} X\right\} \leqq \rho(x, t) \leqq \\
\\
\quad \bar{\rho}_{0} \exp \left\{\frac{1}{\mu}\left|\rho_{0} v_{0}\right|^{(0)} X\right\}, \\
\left|v_{x}(x, t)\right| \leqq K\left(\left\|v_{0}\right\|^{(1)},\left\|\rho_{0}\right\|^{(1)}, \frac{1}{\bar{\rho}_{0}}\right)<+\infty,
\end{array}\right.
$$

where $K$ increases as each argument increases.

Remark. (i) The word "regular" means, exactly speaking, regular up to the boundary.

(ii) If there exists a regular solution $(v, \rho)$ defined in $[0, X] \times[0, \infty)$, then $(v, \rho) \in H_{T}^{2+\alpha} \times B_{T}^{1+\alpha}$ for an arbitrary $T \in(0, \infty)$. 


\section{References}

[1] Bateman, H., Some recent rescarches on the motion of fluids, Monthly Weather Revicw, 43 (1915), 163-170.

[2] Burgers, J., A mathematical model illusilating the theory of turbulence, Advanced in Applied Mechanics, Academic Press̄, (1948).

[3] Cole, J. D., On a quasi-linear parabolic equation occuiring in aerodynamics, Quart. Appl. Math., 9, 3 (1951), 225-236.

[4] Эйдельман, С. 」., Параболические системы. Hayka, (1964).

[5] Friedman, A., Partial differential equations of parabolic type. Prentice Hall, (1964).

[6] Hopf, E., The partial differential equation $u_{t}+u u_{x}=\mu u_{r x}$, Comm. Pure Appl. Math., 5 (1952), 201-230.

[7] Itaya, N., The existence and uniqueness of the solution of the equations describing compressible viscous fluid flow, Proc. Japan Acad., 46, 4 (1970), 379-382.

[8] Itaya, N., On the Cauchy problem for the system of fundamental equations describing the movement of compressible viscous fluid, Ködai Math. Sem. Rep., 23, 1 (1971), 60-120.

[9] Itaya, N.. A survey on two models of the system of equations for compressible viscous fluid, Jimmon-ronshî of Kôbe College of Commerce, 7, 3-4 (1972), 89-95, (Japanese).

[10] Jtaya, N., On the uniqueness of the solution of the generalized Burgers' equation, et al., Ibid. 8, 4 (1973), 1-13. (Japanese).

[11] Itaya, N., On the temporally global problem of the generalized Burgers' equation, J. Math. Kyoto Univ. 14, 1 (1974), 129-177.,

[12] Itô, S., Partial diffcrential equations, Baihûkan, (1966), (Japanese).

[13] Itô, S., Fundamental solutions of parabolic differential equations and boundary value problems, Japan J. Math., 27 (1957), 55-102.

[14] Іадыжегская, О. А., Солонников, Н.Н. и Н.Н. Уральцева, Аинейние и квазилинейне уравнения тараболического тила, Наука, (1967).

[15] Олейник, О.А., и Бентцелъ, Т.د., Первая краевая задачаи задача Коши для квазилинейных уравнений тараболического тила, Mam. Cb., 41 (83), 1 (1957), 105-128.

[16] Рождественский, В.А. и Яненко, Н.Н., Системы квазилинейных уравнений, Наука, (1968).

[17] Tani, A., On some remarks of the mixed problem of Burgers' equation, Thesis of Mastership, Tokyo Inst. Tech., (1973), (Japanese). 
